\title{
A general solution to broad-spectrum vaccine design for rapidly mutating viruses
}

Jacob Glanville ( $\nabla$ jake@centivax.com )

Centivax Inc.

\section{Sarah Ives}

Distributed Bio Inc.

Jean-Philippe Bürckert

Centivax Inc. https://orcid.org/0000-0001-7741-1164

\section{Christina Pettus}

Distributed Bio Inc.

\section{Karina Reyna Peñate}

Centivax Inc.

\section{Rachel Hovde}

Distributed Bio Inc.

Nicholas Bayless

Parker Institute for Cancer Immunotherapy

\section{Devanshi Shanghavi}

BioMarin Pharmaceutical Inc.

\section{Keith Glanville}

Distributed Bio Inc.

\section{Erwin Calgua}

University of San Carlos

\section{Sawsan Youssef}

Centivax Inc.

\section{Article}

Keywords: rapidly evolving pathogens, rapid mutation, viruses, broad-spectrum vaccine.

Posted Date: December 29th, 2020

DOl: https://doi.org/10.21203/rs.3.rs-100459/v1

License: (c) (1) This work is licensed under a Creative Commons Attribution 4.0 International License. 



\title{
A general solution to broad-spectrum vaccine design for rapidly mutating viruses
}

Sarah Ives*, Jean-Philippe Bürckert, Christina Pettus, Karina Reyna Peñate, Rachel Hovde, Nicholas L Bayless, Devanshi Shanghavi, Keith Glanville, Erwin Calgua, Sawsan Youssef, Jacob Glanville*+

* these authors contributed equally

+ corresponding author

\begin{abstract}
Rapidly evolving pathogens pose a challenge to vaccine design, as their mutations render previous vaccine responses obsolete. For influenza, conserved epitopes on the viral coat proteins have been identified, but mysteriously they are missed by the antibodies elicited by most vaccine recipients $[1,2]$. In simulated immunizations using 263 million antibodyhemagglutinin ( $\mathrm{HA})$ structural docking solutions, non-conserved epitopes were immunodominant when HAs were immunized at standard concentrations. However, by vaccinating with a pool of 30 diverse and dilute $\mathrm{HA}$ variants, B-cells that recognize broadlyconserved epitopes across HA receive up to 30-fold higher antigen dose, with concentration being linearly correlated to conservation on a per epitope basis. If individual variants are at concentrations below the minimum threshold of immune activation, then cross-reactive B-cells will be preferentially elicited. In pig immunizations, the approach induced a broad-spectrum antibody response against a panel of 36 strains from 1918-2014, including all pandemic strains from the past century and multiple strains not in the vaccine. In further swine studies with a vaccine containing HAs from 1918-2008, we observe broad-spectrum neutralizing responses against 6 future heterologous strains, including pandemic strains, spanning H1N1 2009-2017 and H3N2 2009-2014. Our results support a greater understanding of why non-conserved epitopes are immunodominant, as well as indicate a general solution to overcome this in broadspectrum vaccine design.
\end{abstract}

\section{Full}

B-cells in the adaptive immune system can defend against infection by producing antibodies that specifically bind to pathogen surface proteins. Many pathogens evade this immune response by varying their surface proteins [3]. Viruses including influenza, HIV, coronavirus, Ebola, CMV, herpes, Dengue, Zika, and the common cold accomplish this through mutation [4]. These variations render previous antibody responses obsolete, causing the immune system to remain vulnerable while maturing antibodies to the new threat.

Vaccination enables a host to generate protective antibodies prior to infection $[5,6]$. This is accomplished by exposing the immune system of the host to the antigens of a pathogen, where the pathogen is either incomplete, dead or weakened. The great vaccine successes, including smallpox, polio, measles, mumps, rubella, diphtheria, pertussis, tetanus, viral meningitis, hepatitis $A$ and $B$, and human papilloma virus, have been possible because these pathogens all mutate relatively slowly, thus affording a multi-year window for a single vaccine to match and provide protection to community strains. In contrast, vaccination against rapidly mutating 
pathogens provides diminished protection as the antigens change over time [4]. This has resulted in the requirement for a new influenza vaccine every year, and some years providing poor protection due to incorrect predictions of the viral strains likely to be in circulation the following season [7].

In recent years, it has been discovered that even rapidly mutating pathogens contain some regions of broadly conserved residues on their surface coat proteins [8-10]. Antibodies that bind these conserved sets of residues can confer broad protection against many strains of the virus. Such broadly neutralizing antibodies (bnAbs) demonstrate that it should be possible to develop a broad spectrum vaccine against such viruses. However, efforts to elicit such bnAbs through vaccination have been challenging. In the context of influenza, stabilized "headless" H1N1 HA conserved stem immunogens have been shown to increase some signatures of protection against $\mathrm{H} 1 \mathrm{~N} 1$ and related $\mathrm{H} 5 \mathrm{~N} 1$ variants [11]. HA consensus sequence immunogens have been able to demonstrate protection against wildtype strains, provided that consensus sequences were relatively similar to wildtype variants [12]. Chimeric HAs with altered heads but fixed stems similarly improved titers against the stem [13]. Viral-like-particles (VLPs) presenting a mosaic of multiple strains has shown recent promise [14]. However, to date there does not exist a broad-spectrum influenza vaccine able to provide robust, heterologous neutralization against future strains and future pandemics, despite known bnAb epitopes being present in every immunogen. For all such universal vaccine approaches, the mystery has been this: why do immune systems so often miss these broadly conserved epitopes?

Multiple hypotheses have been put forward, including immune history imprinting of nonconserved memory responses [15], autoreactivity of conserved epitopes [16-18], natural glycan shielding [19], steric hindrance accessing conserved epitopes [15], host IgH allelic variation [20], and inherent poor immunogenicity of the stem [21]. However, an unaddressed question remains: what is the probability of a bnAb? Put another way, how many epitopes are there on the surface of hemagglutinin, what is ratio of conserved epitopes to non-conserved epitopes, and therefore what is the probability that we would hit those conserved epitopes in a vaccination event?

In order to quantify the diversity of epitopes on the surface of hemagglutinin and the distribution of their conservation across influenza evolution, we created a computational model to simulate how many B-cell epitopes there are on a typical vaccine antigen for antibodies to recognize and what the distribution of conservation of those epitopes are across a century of viral evolution. We then used the implications of this model, that a significant contributing reason for why non-conserved epitopes are immunodominant is that they are vastly more abundant than broadly conserved epitopes, to design a new vaccine approach that shifts the distribution of epitope abundance to favor responses to more conserved epitopes. We modeled this approach in-silico, then validated it in-vivo in three separate studies in outbred domestic pigs.

\section{Quantifying the number of unique antibodies elicited after vaccination}


Previous studies have established that adult humans contain a B-cell receptor (BCR) repertoire of approximately 100 million unique BCRs and after influenza vaccination approximately 1,000 unique $B C R s$ are elicited to begin affinity maturation (Fig 1A, 1B) $[22,23]$. Serological analysis indicates that approximately $10 \%$ convert efficiently to productive plasma cells, resulting in a flu shot providing serological protection with about 100 unique antibodies (Fig 1C) [24].

\section{Quantifying the number of unique epitopes on hemagglutinin}

Human antibodies typically contact 9-19 residues on the antigens they recognize [25] [26] and mutagenesis studies have demonstrated that only a subset of contact positions form the "critical epitope" that is consequential for maintaining recognition [27]. In order to generate a comprehensive database of unique potential critical epitopes on the surface of the influenza hemagglutinin protein (HA), we performed deep computational protein-protein docking of 559 crystallized human antibody structures against a representative HA structure (3FKU) using ZDOCK [28] (Methods 1.1-1.3). This resulted in 3,718,346 unique contact residue sets (Fig 1D, see Methods 1.4). For every unique contact residue set, we then performed repeat random subsampling of 14 positions that could form a potential critical epitope, resulting in a database of 263,022,674 unique 14-residue potential critical epitopes that could each define the binding determinant of a different antibody (Fig 1E, Methods 1.5).

\section{Quantifying amino acid conservation of all hemagglutinin epitopes}

We next analyzed the conservation of all 263 million potential critical epitopes across a panel of 82 representative $H A$ protein sequences from subtypes $\mathrm{H} 1, \mathrm{H} 2, \mathrm{H} 3, \mathrm{H} 5$, and $\mathrm{H} 7$, and strains spanning the years 1918-2018 (Methods 1.6). The majority of epitopes were seasonal specific, recognizing less than a third of strains within their own subtype, and $24.8 \%$ of $\mathrm{H} 1$ and $50.4 \%$ of $\mathrm{H} 3$ epitopes only being found in 1-3 strains in the database. The analysis identified less than three in ten million HA critical epitopes are universally conserved. About one in a million potential critical epitopes were $95 \%$ conserved across all strains, while $2.3 \%$ of $\mathrm{H} 1$ and $5.7 \%$ of $\mathrm{H} 3$ epitopes were universally conserved within subtypes, and $3.8 \%$ of $\mathrm{H} 1$ and $7.3 \%$ of $\mathrm{H} 3$ epitopes were 95\% conserved within subtype (Fig 1F). Epitopes were overwhelmingly subtype specific: less than $1 \%$ of antibodies raised against $\mathrm{H} 1$ would hit epitopes that cross to $\mathrm{H} 5$, and only $0.0021 \%$ expected to cross to $\mathrm{H} 3$. The resulting distributions suggest that non-broadly conserved epitopes are immunodominant by virtue of vastly outnumbering broadly conserved epitopes. Given 100 responding antibodies, most subjects are not predicted to elicit antibodies against universally conserved epitopes, and even the broadly conserved within-strain epitopes will represent a minority of their serological response. A vaccine that would protect against future pandemic strains must therefore not only elicit one bnAb but elicit bnAbs as a majority of the immune response.

\section{Modeling breadth responses to vaccination}

Using the model, we next asked what breadth of response would be anticipated given various vaccination strategies, defined as percentage of epitopes retained between the immunogens and a panel of 82 HAs spanning 1918 to 2018. In the model, an H1N1 2007 immunization generates epitopes that are over $50 \%$ retained back to 1999 and $96 \%$ retained to 2008 , but drop 5-fold to 20\% retained by H1N1 2009 and other future strains (Fig 1G, Methods 1.7). The 
model results are significantly predictive of in-vivo immunizations of 7 pigs against H1N1 2007 $\left(R^{2}=0.548, p<=0.001 ;\right.$ Methods 1.7-1.8). A comparison of in-vivo immunization of H3N2 1997 to model predictions yielded similarly significant results $\left(R^{2}=0.596, p<=0.001\right.$; Methods 1.7-1.8). In the model, a 1918-2005 COBRA consensus H1N1 immunogen [12] shared over $50 \%$ of epitopes with historical H1N1s from 1986-1993, but less than 16\% of epitopes crossed to post-2008 H1N1 strains. A H1N1 2007 HA stabilized stem [11] immunization approach improved the relative proportion of conserved epitopes, but $56 \%$ of epitopes were sterically impeded by the native HA head on live virus or recognize epitopes with residues altered by the stabilization process, thus over half of the serum response against "headless" HA immunogens would not be predicted to contribute to protection (Methods 1.7-1.8).

\section{Increasing average epitope breadth through Conservation-Concentration Coupling}

Given this model, we next asked whether we could design a vaccination strategy that shifts the probability distribution of epitope conservation in-vivo to enrich vaccine-elicited antibodies towards conserved epitopes. We hypothesized that it would be possible to selectively reward B-cells that recognize broadly conserved epitopes by coupling the conservation of these epitopes to their concentration in the vaccine formulation (Conservation-Concentration Coupling or C3, Fig $1 \mathrm{H}$ ). By administering a mixture of many (e.g. 30) diverse variants of HA, each individually below the threshold concentration necessary to elicit a strain-specific immune response, we would effectively remove the dominant population of single variant epitopes from the distribution (Fig 1F), while shared epitopes would be at higher concentration as they were found across multiple HA vaccine components. Thus, this would selectively favor B-cells that recognize shared epitopes across components, with a broadly reactive $B$-cell receiving up to a 30 -fold higher dose than a strain specific B-cell (Fig $1 \mathrm{H}$ ).

We tested this in our model in-silico, immunizing with $30 \mathrm{HAs}$ spanning 1918-2008. As we progressively lowered the concentration of individual antigens to be a dilution factor of $1 / 2$, $1 / 3,1 / 4 \ldots .1 / 10$ th of the threshold concentration $C_{t}$, we observed a progressive increase in breadth of the remaining B-cell epitopes in the distribution, and a notable prediction that as dilution factor increased above 6 , such a low-concentration ensemble would be predicted to elicit a majority of an immune response with cross-reactivity against "future" heterologous viruses, including pandemic H1N1 2009 (Fig 1G, 1H, 1I, Methods 1.9, 2.1).

\section{Conserved Epitope Focusing with Conservation-Concentration Coupling (C3)}

To test our hypothesis, we set out to provide evidence for the following questions:

1) Is there a threshold concentration $C_{t}$ below which a given antigen does not induce an adaptive antibody immune response?

2) Can we combine $N$ sequence-diverse antigens each at a dilution factor $d$ below $C_{t}$ such that only shared epitopes $\varepsilon$ would be presented at an epitope concentration $C_{e}$ above $C_{t}$ ? 


$$
C_{\varepsilon}=\sum_{n=1}^{N} \frac{C_{t}}{d} \begin{cases}N_{n} \subset \varepsilon & 1 \\ \text { else } & 0\end{cases}
$$

3) Can such a formulation as determined in 2) elicit an immune response even though each of $N$ antigens is individually below $C_{t}$ ?

4) Can such a formulation as determined in 2) elicit a more broad immune response than that observed against a single antigen above $C_{t}$ ?

5) Can such a formulation elicit broadly neutralizing titers against future heterologous viruses and pandemic strains?

6) Can such a formulation elicit broadly neutralizing HA monoclonal antibodies against future heterologous viruses and pandemic strains?

Accordingly, we set up three in-vivo experiments using domesticated outbred pigs (Sus scrofa), a model organism and a veterinary target organism for influenza $A$ infection and vaccination (Methods 2.2-2.4).

For in-vivo study \#1, 35 pigs were split into 5 cohorts with 7 pigs per cohort. Each C3 formulation contained a diverse set of $30 \mathrm{HA}$ variants $(\mathrm{H} 1, \mathrm{H} 2, \mathrm{H} 3, \mathrm{H} 5, \mathrm{H} 7$ spanning 1918-2014). Cohorts consisted of a bivalent formulation (BIV, 2 HA antigens H1N1 2007 and H3N2 1997 at 5,000ng/antigen), C3-500 (30 HA antigens at 500ng/antigen), C3-100 (30 HA antigens at 100ng/antigen), C3-50 (30 HA antigens at 50ng/antigen), and vehicle control (PBS + squalene). The animals received 3 vaccinations each 3 weeks apart, with sera collection 3-4 weeks after each vaccination (Days 29, 50, 71). RNA was isolated from PBMCs collected 7 days after the $3^{\text {rd }}$ vaccination (Day 50).

In the second and third in-vivo studies, we removed all HA representatives after 2008 in order to create a "pre-2009" C3 formulation for examining serological response to de facto future viral strains and HAs including pandemic H1N1 2009 through H3N2 2018. A 2008 seasonal bivalent control was similarly formulated, using H1N1 2007 and H3N2 2007. As pigs are not susceptible to $\mathrm{HAB}$ infection, no $\mathrm{HAB}$ antigens were included in either bivalent control or $\mathrm{C} 3$ formulations.

For study \#2, 20 pigs were separated into 5 cohorts with 4 pigs per cohort. Cohorts consisted of BIV (2 HA antigens at 5,000ng/antigen), C3-50 (27 HA antigens at 50ng/antigen), single lowdose H1 (H1N1 2007 at 50ng), single low-dose H3 (H3N2 2007 at 50ng), and vehicle control (PBS + squalene). Cohorts for single HAs administered at low dose (50ng) were included to validate a working $C_{t}$ in pigs. The animals received 6 vaccinations each 21 days apart. Additionally, 3 cohorts (BIV, C3-50, vehicle control) received a $7^{\text {th }}$ vaccination 9 weeks after $6^{\text {th }}$ vaccination. For C3-50, the $7^{\text {th }}$ vaccination was a high-dose boost (27 HA at 500ng/antigen). Sera were collected immediately prior to each vaccination on the same day. Additional sera were collected 4 weeks after the $6^{\text {th }}$ vaccination (Day 135) and 4 weeks after the $7^{\text {th }}$ vaccination (Day 195). RNA was isolated from PBMCs collected 7 days after the $6^{\text {th }}$ vaccination (Day 112). 
For study \#3, 25 pigs were separated into 5 cohorts with 5 pigs per cohort. Cohorts consisted of BIV (5,000ng/antigen) + squalene, C3-50 + squalene, C3-50 + alum, C3-50 + TLR agonists (MPLA, imiquimod), and vehicle control (PBS + squalene + alum + MPLA + imiquimod, Methods 2.2). The animals received 3 vaccinations each 21 days apart, with sera collection immediately prior to each vaccination on the same day. Additional sera were collected 4 weeks after the $3^{\text {rd }}$ vaccination (Day 70). All 3 C3-50 cohorts received $28 \mathrm{HA}$ antigens at 50ng/antigen for the first two vaccinations, and 500ng/antigen for the $3^{\text {rd }}$ vaccination.

\section{Conservation-Concentration Coupling promotes heterologous responses when administered below the threshold concentration $C_{t}$ of individual member antigens}

We established that a 50ng dose of $\mathrm{HA}$ is under the threshold of immune activation by administering a single antigen, either H1N1 2007 or H3N2 2007, at 50ng with 6 total doses each 21 days apart to pigs (study \#2). In both cases, a sera response by ELISA was not detected to either antigen 28 days after the $6^{\text {th }}$ vaccination, confirming the sub-threshold dose (Fig $2 \mathrm{~A}$, Methods 2.5). However, when the two antigens are administered in combination with 25 other HAs spanning 1918-2008, also each at 50ng (C3-50), a sera response to both antigens was detected. Additionally, C3-50 immunization resulted in sera responses to other HAs in C3 and a panel of "future" post-2008 strains not included in the formulation, including H1N1, H3N2, H5N1, and H7N9, spanning 2009-2013. Thus, we demonstrate that a low-dose ensemble can elicit an immune response even though all individual components are below their threshold dose. The results support our C3 model analysis: that in a pool of 27 antigens at individually sub-physiological doses, shared epitopes will be at up to a 27-fold higher dose, and therefore will be successfully targeted and will result in preferentially broad responses that cross to heterologous strains.

\section{Conservation-Concentration Coupling responses are primarily subtype-specific and require a minimum total subtype antigen concentration}

To further explore the relationship between concentration, conservation and immune response breadth, we separated the analysis of C3-elicted binding response by $\mathrm{HA}$ subtype (Fig $2 \mathrm{~B}$ ). The C3-50 formulation in study \#1 contained $10 \mathrm{H} 1 \mathrm{~s}, 7 \mathrm{H3s}, 5 \mathrm{H} 5 \mathrm{~s}, 3 \mathrm{H} 7 \mathrm{~s}$, and $2 \mathrm{H} 2 \mathrm{~s}$, each individually at 50ng. Serum responses were correlated to total subtype dose, with $\mathrm{H} 1$ and $\mathrm{H} 3$ providing the strongest responses, $\mathrm{H} 5$ providing a weaker response, and $\mathrm{H} 2$ and $\mathrm{H} 7$ appearing poorly responded to by C3-50. These results support predictions of the model that responses in one subtype do not necessarily elevate responses to other subtypes, suggesting that broad intra-subtype responses would be much more common than fully universal antibodies (Fig 1F, 2B). The data suggest HA C3 immunization is most effective with a clade dose of 7 or more members (i.e. 350ng of the most conserved epitopes from that clade).

Conservation-Concentration Coupling induces broad reactivity with an inverse dose response Our model predicts that lowering the dose for each individual component in a C3 cocktail should increase the pressure to target only conserved epitopes and thus provide greater breadth of serological response (Fig $1 \mathrm{H}, 1 \mathrm{I})$. To evaluate the relationship between dose and response of $\mathrm{C} 3$, three dosage cohorts of $\mathrm{C} 3$ were tested alongside BIV and vehicle control groups. Sera responses from each of 7 pigs per cohort (study \#1) to a panel of 36 antigens 
indicate that the lowest dose of $\mathrm{C} 3(50 \mathrm{ng} / \mathrm{HA})$ provided the highest serological response to the most antigens, spanning H1N1, H1N3, H2N2, H3N2, H5N1, H6N2, H17N10 from 1918-2014 (Fig 2C). This inverse dose-response is in accordance with the model's predictions, and a unique property of this vaccination strategy (Fig $1 \mathrm{H}, 1 \mathrm{l})$. All three $\mathrm{C} 3$ formulations produced an improved median serum response compared to our bivalent formulation as measured by ELISA (Fig 2C). Additionally, we observed the highest fold-change in sera titer over time, from 7 days to 28 days post- $3^{\text {rd }}$ vaccination, in the lowest dose C3 cohort (Fig 2D). When analyzing responses on a per-cohort, per-animal basis, we observe more animals responding more broadly to more hemagglutinins, with the lowest dose (C3-50ng/antigen) exhibiting greatest breadth (Fig 2E). When analyzed on a per-strain basis, we found C3-50 to elicit a response greater than bivalent positive control (BIV) on 19 strains, including 15 statistically significant strains in the C3 formulation and 4 heterologous strains not in C3 (Fig 3A). Collectively, our data show that breadth of the response is coupled to the concentration as expected by the model, and that as a corollary the $\mathrm{C} 3$ formulation at the lowest dose of 50ng/antigen provides the broadest serum response in the most pigs.

\section{Conservation-Concentration Coupling elicits heterologous neutralization of future and pandemic viruses}

Next, we demonstrate that Conservation-Concentration Coupling elicits robust in-vitro neutralizing serological response to a panel of 8 viruses from 1934-2015, including "future" H1N1 and H3N2 strains spanning 2009-2015 not present in the 2008 C3 formulation, including neutralization of pandemic H1N1-2009 (Fig 3B, 3C, Methods 3.1). Pigs that received C3 (50ng/antigen $\times 27$ antigens, study \#2) neutralized more strains with more pigs responding compared to pigs that received BIV (H1N1 2007 and H3N2 2007, each at 5,000ng), single H1 at 50ng, single $\mathrm{H} 3$ at 50ng, and vehicle control cohorts (Fig 3B). BIV cohort were potently neutralizing their two respective viruses but failed to produce strong neutralizing titers to future H1N1 strains, or H3N2 strains after 2011 (Fig 3C), consistent with the model's predictions. Interestingly, some pigs that received 6 vaccinations of H3N2 2007 at 50ng/dose produced low but detectable neutralization of some H3N2 viral strains from serum 13 weeks after the final $\left(7^{\text {th }}\right)$ vaccination (Fig 3B), despite the absence of detectable binding by ELISA from sera isolated 4 weeks after the 6 th vaccination (Fig 2A). This suggests that our sub-threshold dose of 50ng resulted in a limited delayed response, where the immunization was defective but not entirely inactive given a large number of boosts.

In a third animal study, we confirmed broad neutralization to future viruses while assessing different adjuvants with C3 (50ng/antigen x 28 antigens, study \#3). Again as in study \#2, the 2008 seasonal BIV cohort (H1N1 2007 and H3N2 2007, each 5,000ng + squalene) failed to produce neutralizing titers to 3 "future" post-2008 H1N1 viruses spanning 2009-2017. After 3 vaccinations, C3+alum elicited the highest neutralizing titers in all future viruses 2009-2017 across all pigs in the cohort, followed by C3+squalene (Fig 3D). C3+MPLA+imiquimod did not produce detectable neutralizing titers in future viruses. These results further indicate that the breadth and future neutralizing potential of these vaccines are attributable to the ensemble and not to a specific adjuvant. Collectively our data show that C3 formulations administered at $50 \mathrm{ng} /$ antigen produce sera with broadly neutralizing capacities at a similar level to that of a 
bivalent formulation while also neutralizing a broader range of "future" viruses that emerge up to a decade of viral evolution after those that were included in the antigen formulation.

\section{Conservation-Concentration Coupling induces uniform B cell expansion, somatic hypermutation, antigen-specific and broadly neutralizing pig monoclonal antibodies}

To evaluate the molecular characteristics of the antibodies responding to C 3 immunizations, we isolated antibody repertoire cDNA from PBMCs from all C3-immunized pigs from study \#1 and study \#2. For study \#1, we displayed the pig repertoires on phage display to recover a panel of anti-HA immunogen-specific pig antibodies from multiple animals from all dosage groups (Fig 4A, Methods 4.1-4.3). The antibodies were diverse across animals, were confirmed by ELISA to be specific to HA immunogen, and the majority had undergone somatic hypermutation (SHM) and class-switched to IgG, indicating a normal B-cell memory maturation path for these clones (Fig 4A, Methods 4.1-4.3). We next analyzed the pigs' IgM and IgG B-cell receptor (BCR) repertoires from PBMCs (study \#2, day 112). We observed comparable SHM in the top 100 most expanded clones compared with bivalent (BIV) immunized animals (Fig 4B) consistent with monoclonal observations from study \#1 (Fig 4A). In contrast with the high variability in clonal expansion observed among pigs receiving either bivalent or vehicle immunization schedules, we observed a significantly more uniform BCR repertoire clonotype expansion in the pigs receiving the C3-50 formulation (Fig 4C). Thus, C3 appears to generate a more uniform, and therefore potentially more predictable, immune response.

\section{Conservation-Concentration Coupling elicits potent broadly neutralizing monoclonal antibodies}

To determine whether C3 immunization was able to provoke individual broadly neutralizing monoclonal antibodies, pig monoclonals were isolated from animals from study \#1 by repertoire sequencing and phage display, expressed recombinantly, and screened for binding and breadth against multiple H1N1 and H3N2 hemagglutinins (Methods 4.1-4.3). Pig monoclonal $9 \mathrm{C5}$ was isolated from Pig 25, which received the lowest dose of C3 (50ng/antigen $\mathrm{x}$ 30 antigens Fig 2E). 9C5 shows broad in-vitro neutralization capability against 3 H1N1 and 2 H3N2 viruses spanning 2009-2017 (Fig.4D, 4E). Neutralizing each virus at the lowest antibody dilution tested (each $<0.188 \mathrm{ug} / \mathrm{mL}$ ), $9 \mathrm{C} 5$ more potently neutralizes all H1N1 and H3N2 viruses tested than any of the 3 control broadly neutralizing antibodies CR9114, F10, and C05. Taken together, these results demonstrate that low-dose C3 vaccination not only elicits broadly neutralizing serum titers but also in those same animals we can recover broadly neutralizing monoclonal antibodies, including antibodies capable of neutralizing group 1 and group 2 influenza viruses.

\section{Summary and conclusions}

While multiple other mechanisms may also be acting to drive subdominance of conserved hemagglutinin epitopes, we were able to induce broadly neutralizing responses just by increasing relative frequency of rare but conserved native epitopes. Here we present a model that provides a quantitative explanation that contributes to our understanding of nonconserved epitopes are immunodominant. The model makes the case that the phenomenon of immunodominance of non-conserved epitopes on influenza A can be attributed to there being 
vastly more mutable epitopes than conserved ones. Using this model, we develop conservationconcentration coupling, a novel vaccination approach capable of eliciting broadly reactive and broadly neutralizing anti-influenza antibodies against a century of viral evolution. Providing a higher vaccine dose of highly conserved regions solely by limiting dilution of a diverse pool of vaccine components bypasses the need to perform any special engineering to the components or to know the location of conserved regions on the antigen. This approach is thus generalized and may be applied to other mutating or diverse viruses such as coronaviruses, HIV, HCV, Zika and Dengue, thus providing a new tool to address the limitations of contemporary vaccine design against these pathogens. 


\section{Figure Legends}

Figure 1. Modeling antibody breadth probabilities elicited by four hemagglutinin vaccine design strategies.

Approximately A) 100 million unique B-cell receptors exist in a human adult, B) 1000 unique B cell lineages are elicited by influenza vaccination, and C) 100 unique serum antibodies form influenza vaccine responses. D) in-silico deep repertoire docking of 559 crystallized human antibodies against HA has identified 3.7 million unique potential antibody docking geometries on the surface of HA. E) Stochastic repeat subsampling of 14 residues from each docking solution resulted in a database of 263 million unique potential critical B cell epitopes on HA. and a rarefaction trend to an excess of 1 billion epitopes. F) Less than $10 \%$ of epitopes are highly conserved within strain, and less than $0.0001 \%$ are universal. G) Proportion of shared epitopes between immunization agent and H1N1 variants from 1918-2017, for four vaccine design strategies: H1N1 2007, stem-stabilized H1N1 2007, COBRA consensus, and C3 1918-2007 at $\mathrm{C} 3$ threshold concentration $\mathrm{C}_{\mathrm{t}}=1 / 2-1 / 10$. H) Co-administration of HA from many strains preferentially doses B-cells that recognize more broadly conserved epitopes (indicated as "bnAb") via "Concentration-Conservation Coupling" (C3). I) Future heterologous breadth of epitopes elicited modeled as a function of $\mathrm{C} 3$ threshold concentration $\mathrm{C}_{\mathrm{t}}$. 
Figure 2. Conservation-Concentration Coupling promotes heterologous responses to HAs spanning 1934-2014 when administered below the threshold dose of individual member antigens.

A) ELISA signals of individual pig sera (study \#2, Day 135) to $10 \mathrm{HA}$ antigens ( $\mathrm{H1}, \mathrm{H3}, \mathrm{H} 5, \mathrm{H7}$ spanning 1934-2013) across 4 cohorts (each $n=4$ ) of vehicle control, 50ng H1N1 2007, 50ng H3N2 2007, and C3-50 (27 HAs at 50ng/HA). Box and whiskers plots show median and IQR; whiskers show $1.5 \times$ IQR of the lower and upper quartiles. Points represent outliers. Orange indicates "future" HAs not present in the 1918-2008 C3 vaccine formulation. B) ELISA signals from pigs of the C3-50 cohort (study \#1, Day 71) are shown separated into the different subtypes present in the $\mathrm{C} 3-50$ formulation $(\mathrm{H} 1, \mathrm{H} 2, \mathrm{H} 3, \mathrm{H} 5$ and $\mathrm{H} 7)$. The $X$-axis shows the number and amount of HAs per subtype. Pearson's correlation coefficient for number of HA antigens vs ELISA signal is shown as $\mathrm{R}^{2}$ with corresponding $\mathrm{p}$-value. C) Average ELISA signals per pig across all tested strains ( $n=36$, spanning $\mathrm{H} 1, \mathrm{H} 2, \mathrm{H} 3, \mathrm{H} 5, \mathrm{H} 7$ and $\mathrm{H} 17$ from 1918-2014) per pig (study \#1, Day 71), grouped by cohort. D) Fold-change of average ELISA signal per pig from C) between 7 and 28 days post- $3^{\text {rd }}$ vaccination (Days 50,71 ). E) Heatmap of ELISA signals (\% max $\mathrm{OD}_{450 \mathrm{~nm}}$ ) from individual pig sera of 5 immunization cohorts (study \#1, Day 71) binding against $36 \mathrm{HAs}(\mathrm{H1}, \mathrm{H} 2$, H3, H5, H6, H7, H17 spanning 1918-2014) and bnAb controls (C05, F10, CR9114). Heatmap coloring was adapted using quantile breaks to adjust for the ELISA data distribution. Positive strain coverage was determined as percent values above average of the vehicle control $+3 \times$ SD for each strain.

B, C, D) Box and whiskers plots show median and IQR; whiskers show $1.5 \times$ IQR of the lower and upper quartiles. $P$ values are calculated using Kruskal-Wallis rank sum test with Dunn's correction for multiple comparisons. Selected $P$ values are reported as adjusted and indicated as $* \mathrm{P}<0.05, * * \mathrm{P}<0.01, * * * \mathrm{P}<0.001, * * * * \mathrm{P}<0.0001$. 
Figure 3. Conservation-Concentration Coupling elicits heterologous binding and neutralization of future and pandemic viruses

A) ELISA signals of pig sera to $36 \mathrm{HA}$ antigens ( $\mathrm{H1}, \mathrm{H2}, \mathrm{H3}, \mathrm{H5}, \mathrm{H6}, \mathrm{H7}, \mathrm{H} 17$ spanning 1918-2014) across immunization cohorts (each $n=7$ pigs) of C3-50, BIV cohort and vehicle control (study \#1, Day 71). Bars represent mean across all pigs of a cohort; error bars indicate positive standard deviation. Orange bars indicate HAs not present in the C3 vaccine formulation. Asterisks indicate statistically significant differences between C3-50 and bivalent formulation with adjusted $p$ values $<0.05$ or lower. $P$ values were determined using strain-wise Wilcoxon Rank Sum test with alternative hypothesis of BIV signals being less than C3-50 signals. Benjamini Hochberg correction was applied to adjust for multiple testing. B) Neutralization breadth is shown as average number of strains neutralizing per pig by cohort and as number of positive tests per cohort are shown as scatterplot. Strains tested are shown in 1C. Sera were not pooled (study \#2, Day 195). Cohorts were vehicle control, seasonal BIV (H1N1 2007 and H3N2 2007 each at 5,000ng), low-dose H1N1 2007 at 50 ng, low-dose H3N2 2007 at 50 ng, and C3-50 (27 HAs at 50ng/HA). Error bars indicate standard deviation. C) Neutralization strength for study \#2 is reported as reciprocal neutralizing dilution per strain across cohorts using bar charts. Bars indicate the mean across all pigs $(n=4)$ of a cohort and error bars indicate standard deviation. "Future" strains tested that are not part of the 2008 C3 formulation are shown in orange. D) Neutralization strength for study \#3 (Day 70) is reported as reciprocal neutralizing dilution per strain across cohorts using bar charts. Bars indicate the mean across all pigs $(n=5)$ of a cohort and error bars indicate standard deviation. Points show the individual value per pig. "Future" strains tested that are not part of the 2008 C3 formulation are shown in orange. Cohorts were seasonal BIV (H1N1 2007 and H3N2 2007 each at 5,000ng + squalene), and 3 cohorts of C3-50 (28 HAs at 50ng/HA), with squalene, TLR-agonists, and alum adjuvants, respectively. 
Figure 4. Conservation-Concentration Coupling induces uniform B cell expansion, affinity maturation, immunogen-specific and broadly neutralizing monoclonal antibodies

A) Pig monoclonal antibodies specific to $\mathrm{HA}$ immunogen recovered from all $\mathrm{C} 3$ dosage groups (50ng/ag, 100ng/ag, 500ng/ag) of study \#1. Clones were recovered by isolating antibody repertoire DNA from PBMCs from all C3 immunized animals from study \#1, then selecting for anti-HA binder by phage display, and confirming individual clones by ELISA. B) Somatic hypermutation of top 100 most expanded clones in pigs, by isotype and immunization cohort. Immunization cohorts: a C3 formulation of $27 \mathrm{HAs}$ at 50ng/HA $(n=4)$, a bivalent cohort (H1N1 2007 and H3N2 2007 each at 5,000ng, $n=3$ ) and vehicle control (PBS + squalene, $n=4$ ). Lymphocytes collected 7 days after $6^{\text {th }}$ vaccination (Day 112 , study \#2). C) Percentage of peripheral B-cell receptor repertoire occupied by top $n$ clonotypes across pigs per immunization group. Gray: 95\% confidence interval. D) Minimum antibody concentration (ug/mL) sufficient for broadly neutralizing pig monoclonal antibody 9C5 and controls CR9114, F10, C05 in-vitro neutralization of a panel of 5 Influenza A strains. Clone 9C5 was elicited by C3-50 immunized Pig \#25. E) Sequence alignment of broadly neutralizing pig monoclonal antibody 9C5 to IMGT V Quest pig (Sus scrofa) germline genes. IMGT numbering. Detected somatic hypermutations underlined, CDRH3 marked in bold. 


\section{References}

1. Sui, J., et al., Structural and functional bases for broad-spectrum neutralization of avian and human influenza A viruses. Nat Struct Mol Biol, 2009. 16(3): p. 265-73.

2. Dreyfus, C., et al., Highly conserved protective epitopes on influenza B viruses. Science, 2012. 337(6100): p. 1343-8.

3. Elena, S.F. and R. Sanjuan, Adaptive value of high mutation rates of RNA viruses: separating causes from consequences. J Virol, 2005. 79(18): p. 11555-8.

4. Karlsson Hedestam, G.B., et al., The challenges of eliciting neutralizing antibodies to HIV1 and to influenza virus. Nat Rev Microbiol, 2008. 6(2): p. 143-55.

5. Plotkin, S.A., Immunologic correlates of protection induced by vaccination. Pediatr Infect Dis J, 2001. 20(1): p. 63-75.

6. Amanna, I.J., I. Messaoudi, and M.K. Slifka, Protective immunity following vaccination: how is it defined? Hum Vaccin, 2008. 4(4): p. 316-9.

7. Russell, C.A., et al., Influenza vaccine strain selection and recent studies on the global migration of seasonal influenza viruses. Vaccine, 2008. 26 Suppl 4: p. D31-4.

8. McLellan, J.S., et al., Structure of HIV-1 gp120 V1/V2 domain with broadly neutralizing antibody PG9. Nature, 2011. 480(7377): p. 336-43.

9. Walker, L.M., et al., Broad and potent neutralizing antibodies from an African donor reveal a new HIV-1 vaccine target. Science, 2009. 326(5950): p. 285-9.

10. Binley, J.M., et al., Comprehensive cross-clade neutralization analysis of a panel of antihuman immunodeficiency virus type 1 monoclonal antibodies. J Virol, 2004. 78(23): p. 13232-52.

11. Impagliazzo, A., et al., A stable trimeric influenza hemagglutinin stem as a broadly protective immunogen. Science, 2015. 349(6254): p. 1301-6.

12. Giles, B.M. and T.M. Ross, A computationally optimized broadly reactive antigen (COBRA) based H5N1 VLP vaccine elicits broadly reactive antibodies in mice and ferrets. Vaccine, 2011. 29(16): p. 3043-54.

13. Krammer, F., et al., Chimeric hemagglutinin influenza virus vaccine constructs elicit broadly protective stalk-specific antibodies. J Virol, 2013. 87(12): p. 6542-50.

14. Kanekiyo, M., et al., Author Correction: Mosaic nanoparticle display of diverse influenza virus hemagglutinins elicits broad B cell responses. Nat Immunol, 2019. 20(6): p. 765.

15. Andrews, S.F., et al., Immune history profoundly affects broadly protective $B$ cell responses to influenza. Sci Transl Med, 2015. 7(316): p. 316ra192.

16. Guthmiller, J.J. and P.C. Wilson, It's Hard to Teach an Old B Cell New Tricks. Cell, 2020. 180(1): p. 18-20.

17. Bajic, G., et al., Autoreactivity profiles of influenza hemagglutinin broadly neutralizing antibodies. Sci Rep, 2019. 9(1): p. 3492.

18. Haynes, B.F., et al., Cardiolipin polyspecific autoreactivity in two broadly neutralizing HIV-1 antibodies. Science, 2005. 308(5730): p. 1906-8.

19. Bajic, G., et al., Influenza Antigen Engineering Focuses Immune Responses to a Subdominant but Broadly Protective Viral Epitope. Cell Host Microbe, 2019. 25(6): p. 827-835 e6.

20. Avnir, Y., et al., IGHV1-69 polymorphism modulates anti-influenza antibody repertoires, correlates with IGHV utilization shifts and varies by ethnicity. Sci Rep, 2016. 6: p. 20842. 
21. Tan, H.X., et al., Subdominance and poor intrinsic immunogenicity limit humoral immunity targeting influenza HA stem. J Clin Invest, 2019. 129(2): p. 850-862.

22. Jiang, N., et al., Lineage structure of the human antibody repertoire in response to influenza vaccination. Sci Transl Med, 2013. 5(171): p. 171ra19.

23. Vollmers, C., et al., Genetic measurement of memory B-cell recall using antibody repertoire sequencing. Proc Natl Acad Sci U S A, 2013. 110(33): p. 13463-8.

24. Lee, J., et al., Molecular-level analysis of the serum antibody repertoire in young adults before and after seasonal influenza vaccination. Nat Med, 2016. 22(12): p. 1456-1464.

25. Stave, J.W. and K. Lindpaintner, Antibody and antigen contact residues define epitope and paratope size and structure. J Immunol, 2013. 191(3): p. 1428-35.

26. Potocnakova, L., M. Bhide, and L.B. Pulzova, An Introduction to B-Cell Epitope Mapping and In Silico Epitope Prediction. J Immunol Res, 2016. 2016: p. 6760830.

27. Van Blarcom, T., et al., Precise and efficient antibody epitope determination through library design, yeast display and next-generation sequencing. J Mol Biol, 2015. 427(6 Pt B): p. 1513-1534.

28. Pierce, B.G., et al., ZDOCK server: interactive docking prediction of protein-protein complexes and symmetric multimers. Bioinformatics, 2014. 30(12): p. 1771-3. 


\section{Figures}

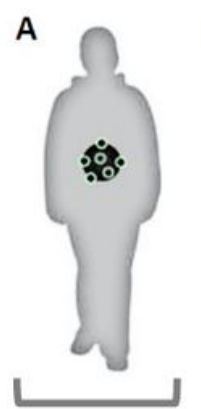

$10^{8}$

B-cell Repertoire Unique BCRs in the antibody repertoire of one adult

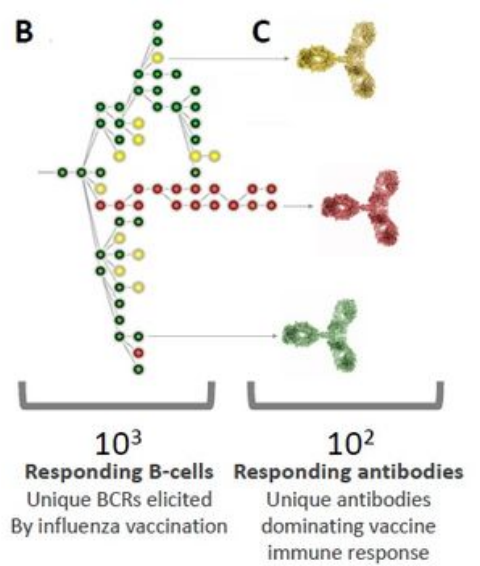

immune response



H

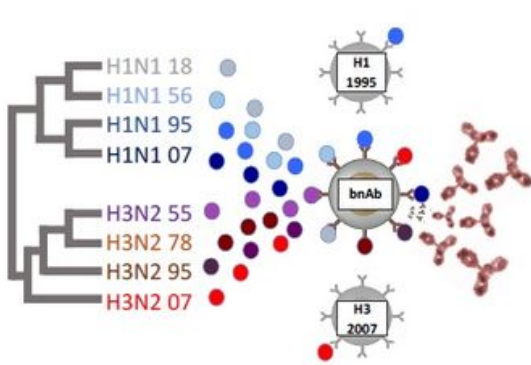

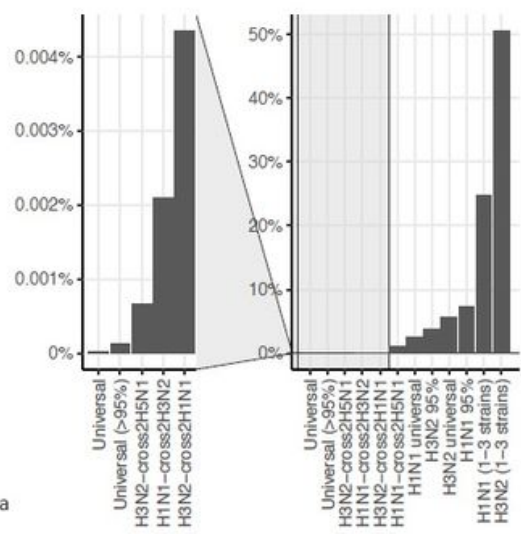

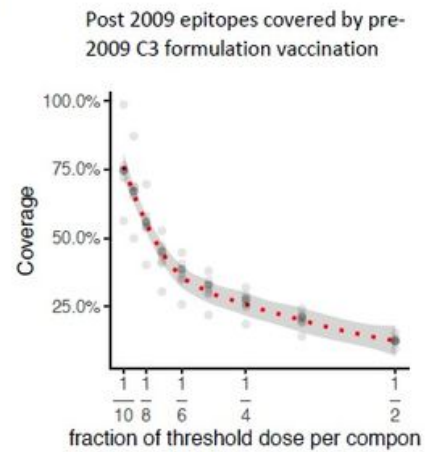

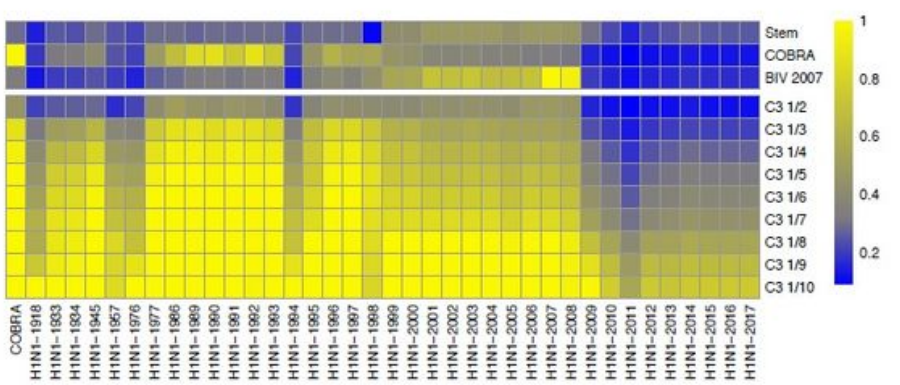

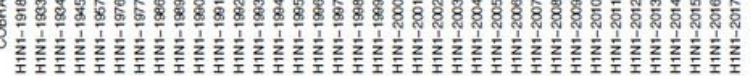

\section{Figure 1}

Modeling antibody breadth probabilities elicited by four hemagglutinin vaccine design strategies. Approximately A) 100 million unique B-cell receptors exist in a human adult, B) 1000 unique $B$ cell lineages are elicited by influenza vaccination, and C) 100 unique serum antibodies form influenza vaccine responses. D) in-silico deep repertoire docking of 559 crystallized human antibodies against HA has identified 3.7 million unique potential antibody docking geometries on the surface of HA. E) Stochastic repeat subsampling of 14 residues from each docking solution resulted in a database of 263 million unique potential critical B cell epitopes on HA. and a rarefaction trend to an excess of 1 billion epitopes. F) Less than $10 \%$ of epitopes are highly conserved within strain, and less than $0.0001 \%$ are universal. G) Proportion of shared epitopes between immunization agent and H1N1 variants from 19182017, for four vaccine design strategies: H1N1 2007, stem-stabilized H1N1 2007, COBRA consensus, and C3 1918-2007 at C3 threshold concentration $\mathrm{Ct}=1 / 2-1 / 10$. H) Co-administration of HA from many strains preferentially doses B-cells that recognize more broadly conserved epitopes (indicated as "bnAb") via "Concentration-Conservation Coupling" (C3). I) Future heterologous breadth of epitopes elicited modeled as a function of $\mathrm{C} 3$ threshold concentration $\mathrm{Ct}$. 
A.

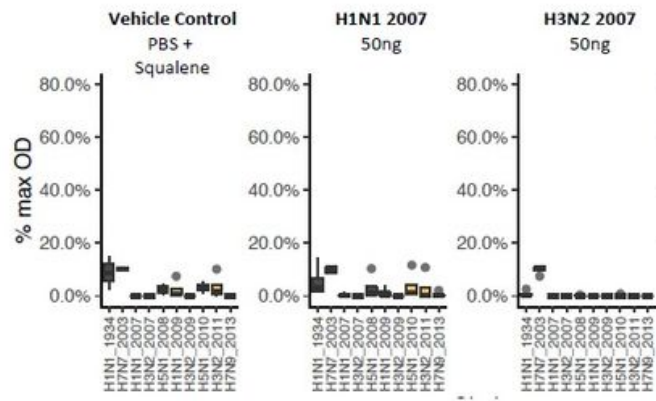

B.

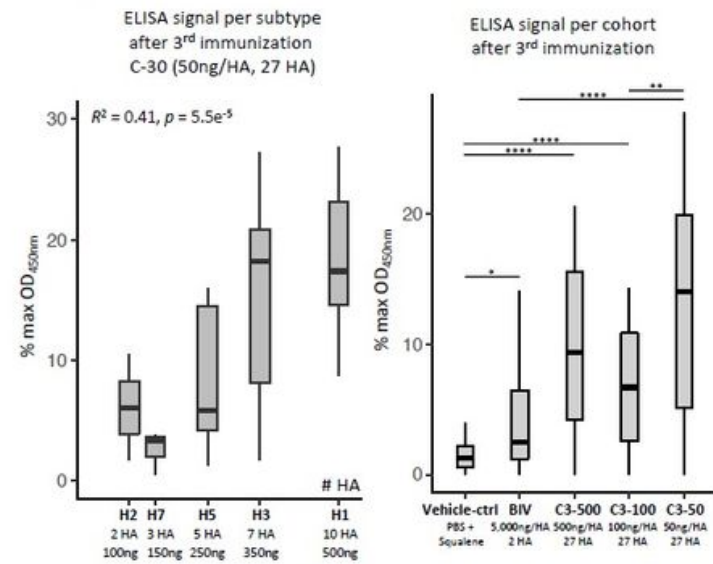

D.

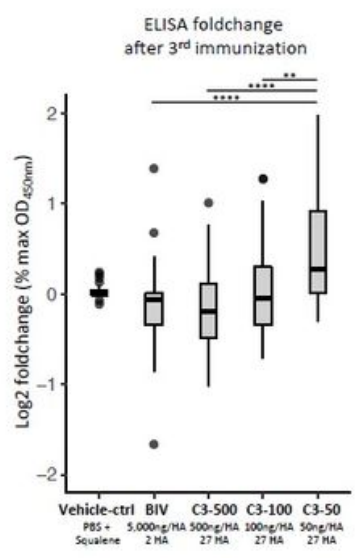

E.

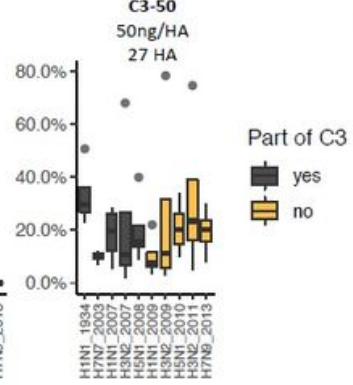

E.
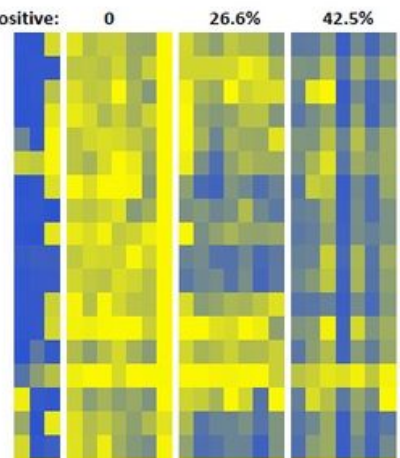

$53.2 \% \quad 62.7 \%$

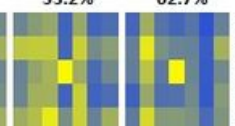

H1N1_1918

H1N1_1933

H1N1_1976

H1N1_1977

H1N1_1991

H1N1_1995

H1N1_1998

H1N1_2006

H1N1_2009

HiN1_2009

H1N3_1976
H2N2_1957

H2N2_2005

H3N2_1968

H3N2_1995

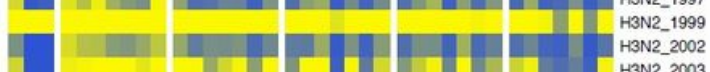

$43 N_{2} 2004$

H3N2_2007

H3N2_2007

H3N2_2011

H5N1_1997

H5N1_2004

H5N1_2008

H5N1_2009

15N1_2010

(1)

H7N7_1975

H7N7_2003

H7N7_2013

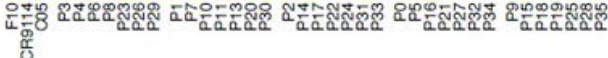

H17N10_2009

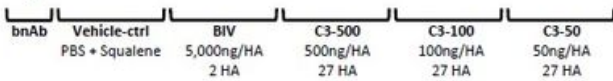

Figure 2

Conservation-Concentration Coupling promotes heterologous responses to HAs spanning 1934-2014 when administered below the threshold dose of individual member antigens. A) ELISA signals of individual pig sera (study \#2, Day 135) to 10 HA antigens (H1, H3, H5, H7 spanning 1934-2013) across 4 cohorts (each $n=4$ ) of vehicle control, 50ng H1N1 2007, 50ng H3N2 2007, and C3-50 (27 HAs at 50ng/HA). Box and whiskers plots show median and IQR; whisker show $1.5 \times$ IQR of the lower and upper quartiles. Points represent outliers. Orange indicates "future" HAs not present in the 1918-2008 C3 vaccine formulation. B) ELISA signals from pigs of the C3-50 cohort (study \#1, Day 71) are shown separated into the different subtypes present in the $\mathrm{C} 3-50$ formulation $(\mathrm{H} 1, \mathrm{H} 2, \mathrm{H} 3, \mathrm{H} 5$ and $\mathrm{H} 7)$. The Xaxis shows the number and amount of HAs per subtype. Pearson's correlation coefficient for number of HA antigens vs ELISA signal is shown as R2 with corresponding p-value. C) Average ELISA signals per pig across all tested strains ( $\mathrm{n}=36$, spanning $\mathrm{H1}, \mathrm{H} 2, \mathrm{H} 3, \mathrm{H} 5, \mathrm{H} 7$ and $\mathrm{H} 17$ from 1918-2014) per pig (study \#1, Day 71), grouped by cohort. D) Fold-change of average ELISA signal per pig from C) between 7 and 28 days post-3rd vaccination (Days 50,71 ). E) Heatmap of ELISA signals (\% max OD450nm) from individual pig sera of 5 immunization cohorts (study \#1, Day 71) binding against $36 \mathrm{HAs}(\mathrm{H1}, \mathrm{H2}, \mathrm{H3}, \mathrm{H} 5, \mathrm{H} 6, \mathrm{H} 7$, H17 spanning 1918-2014) and bnAb controls (C05, F10, CR9114). Heatmap coloring was adapted using quantile breaks to adjust for the ELISA data distribution. Positive strain coverage was determined as percent values above average of the vehicle control $+3 x$ SD for each strain. $B, C, D)$ Box and whiskers plots show median and IQR; whiskers show 1.5 x IQR of the lower and upper quartiles. P values are calculated using Kruskal-Wallis rank sum test with Dunn's correction for multiple comparisons. Selected P

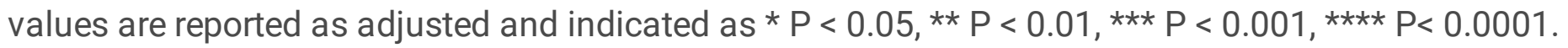



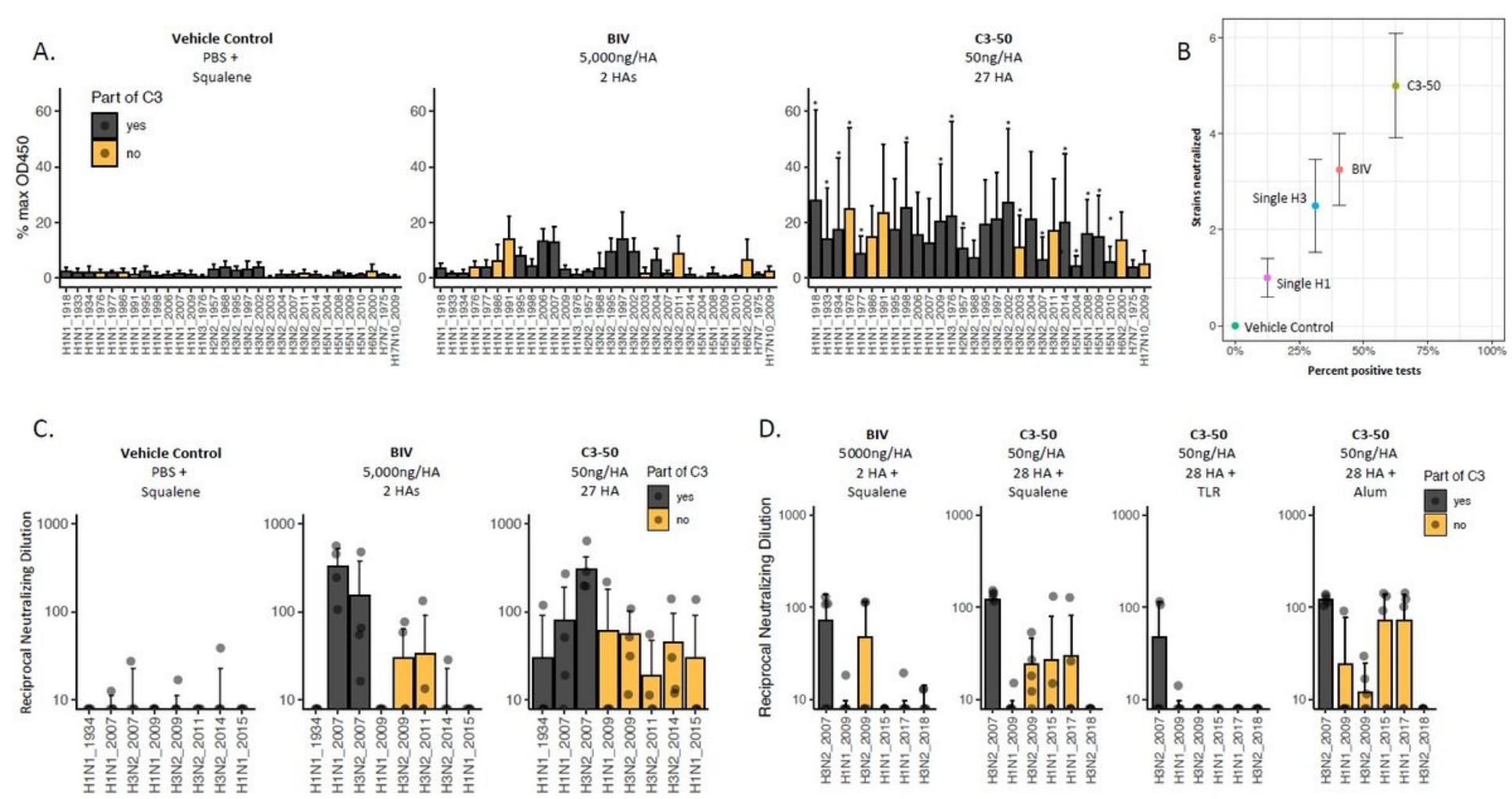

\section{Figure 3}

Conservation-Concentration Coupling elicits heterologous binding and neutralization of future and pandemic viruses A) ELISA signals of pig sera to $36 \mathrm{HA}$ antigens $(\mathrm{H} 1, \mathrm{H} 2, \mathrm{H} 3, \mathrm{H} 5, \mathrm{H} 6, \mathrm{H} 7, \mathrm{H} 17$ spanning 1918-2014) across immunization cohorts (each $n=7$ pigs) of C3-50, BIV cohort and vehicle control (study \#1, Day 71). Bars represent mean across all pigs of a cohort; error bars indicate positive standard deviation. Orange bars indicate HAs not present in the $\mathrm{C} 3$ vaccine formulation. Asterisks indicate statistically significant differences between C3-50 and bivalent formulation with adjusted $p$ values $<0.05$ or lower. $\mathrm{P}$ values were determined using strain-wise Wilcoxon Rank Sum test with alternative hypothesis of BIV signals being less than C3-50 signals. Benjamini Hochberg correction was applied to adjust for multiple testing. B) Neutralization breadth is shown as average number of strains neutralizing per pig by cohort and as number of positive tests per cohort are shown as scatterplot. Strains tested are shown in 1C. Sera were not pooled (study \#2, Day 195). Cohorts were vehicle control, seasonal BIV (H1N1 2007 and H3N2 2007 each at 5,000ng), low-dose H1N1 2007 at 50 ng, low-dose H3N2 2007 at 50 ng, and C350 (27 HAs at 50ng/HA). Error bars indicate standard deviation. C) Neutralization strength for study \#2 is reported as reciprocal neutralizing dilution per strain across cohorts using bar charts. Bars indicate the mean across all pigs $(n=4)$ of a cohort and error bars indicate standard deviation. "Future" strains tested that are not part of the $2008 \mathrm{C} 3$ formulation are shown in orange. D) Neutralization strength for study \#3 (Day 70) is reported as reciprocal neutralizing dilution per strain across cohorts using bar charts. Bars indicate the mean across all pigs $(n=5)$ of a cohort and error bars indicate standard deviation. Points show the individual value per pig. "Future" strains tested that are not part of the 2008 C3 formulation are shown in orange. Cohorts were seasonal BIV (H1N1 2007 and H3N2 2007 each at 5,000ng + squalene), 
and 3 cohorts of C3-50 (28 HAs at 50ng/HA), with squalene, TLR-agonists, and alum adjuvants, respectively.

A

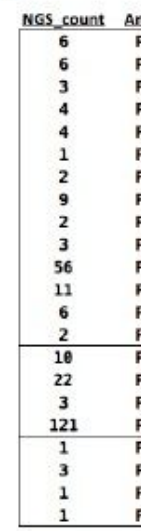

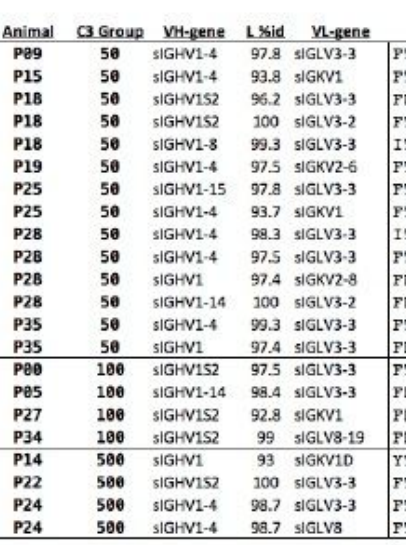

CDR1

TPFITYIN $\quad$ ACISTCGSDTYYA

FTFSSTYIN AGISSGGSDTYYA FDFSDYDF5 AYINTDGGSTYYA $\begin{array}{ll}\text { FTFSSYBIN } & \text { AALDTVGGNTGYA } \\ \text { ITFSTYAVS } & \text { AGIDTGSWSGSZYYA }\end{array}$ FTFSDNYIN AGISSSGSDTYYA FTFSSYGMS AEIRSSGGSTYYA PTFSSUWIH MAISTGGGSTYYA ITFSSTPIT ATITSSGGDTYYA FTFSSTYIN ATISTTGAKTYYA $\begin{array}{ll}\text { FOFSGEAFS } & \text { AAIGNNDDDRATYYA } \\ \text { FDFSDYAFS } & \text { AVISTSGSDZYYA }\end{array}$ $\begin{array}{ll}\text { PTFNTIVIN } & \text { AGISTSGRTTYYA } \\ \text { FDFSSYPIS } & \text { ATISSGGGDAYYA }\end{array}$ \begin{tabular}{ll} 
PDFSSYPIS & ATISSGGGDAYYA \\
\hline FTFSSYPIG & AGIGSSGSGTYYA
\end{tabular} $\begin{array}{ll}\text { FTFSSYPIG } & \text { AGIGSSGSGTYYA } \\ \text { PDFSDYAFS } & \text { AAISPGGSVEFYA }\end{array}$ PDFSDYAFS RAISPGGSVEFYA FOFSDYAFS AAISTSGCDTFYA YTFSDYBIG GHISPGGSIEFYA $\begin{array}{ll}\text { FTFTSYAVT } & \text { AGISSYSVNTYYA } \\ \text { FTFSSTYIN } & \text { AAISTSGGSTYYA }\end{array}$ $\begin{array}{ll}\text { PTFSSTYIN } & \text { AAISTSGGSTYYA } \\ \text { PTFSSTYIN } & \text { AGISSGGSDTYYA }\end{array}$

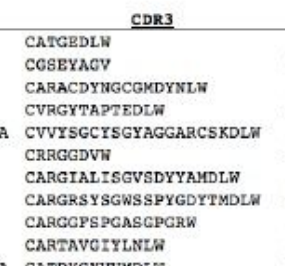

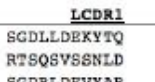
R2SQSVSSELD SGDRLDEVYAR ZGNNTGRKDIQ SGDLLDEKYTR RSDLSLEKYGS RASOSISKYLA SGDLLDEKYTR SCDLLDEKYTO RSSQSLVDSDGDSL RSSQSLVDSDG BGNNTGRKDIQ

CGTGGPTYG

CAVQRLLLRGWAMDLW CGRYGHPDLW CAAGDSWDY CARKGMDLI CSRVSDDLM CAARGAMDLW CARVSLDUF CARAPNCYLYGDTCYD CATAMVLVAMDLN
CKRGIYGGDGDLW SGDLLDKKYTQ
SGDLLDEKYTQ SGDLLDEKYTR SGDLLDEKYTQ RASOSISSYLA AFSSGSVTSSNYPS RAGOSVSDSTA RAGQSVSDNLA SGDLLDEKYTQ
SGDLLDEKYTO AFSSGSVTTMYYP

C3-50 50ng/ HA
$27 \mathrm{HA}$

C Vehicle Control PBS +
Squalene av $5,000 \mathrm{ng} / \mathrm{HA}$
$2 \mathrm{HAs}$ D
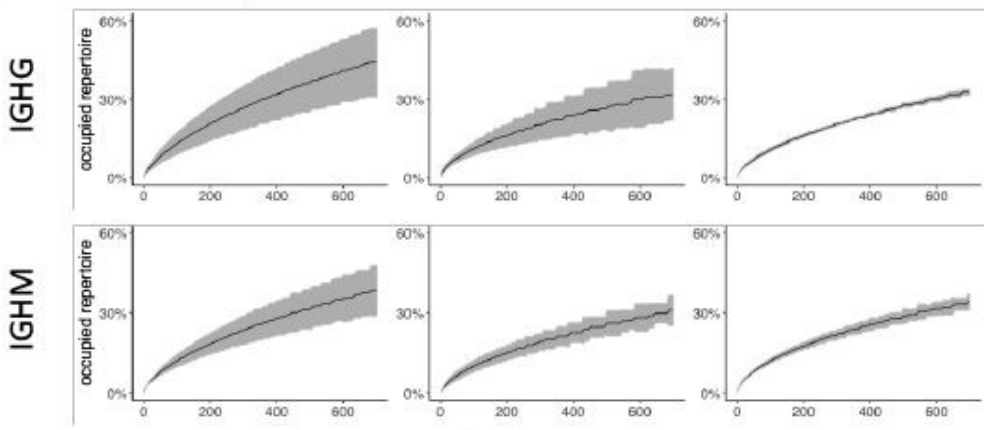

top $n$ clonotypes
Minimum antibody concentration ( $\mathrm{ug} / \mathrm{mL}$ ) sufficient for neutralization

\begin{tabular}{lllll}
\hline Virus / Antibody & $\begin{array}{l}\text { 9C5 } \\
\text { (ug/mL) }\end{array}$ & $\begin{array}{l}\text { CR9114 } \\
\text { (ug/mL) }\end{array}$ & F10 (ug/mL) & C05 (ug/mL) \\
\hline H3N2/Brisbane/2007 & $<0.125$ & 25 & $>12.5$ or $\mathrm{n} / \mathrm{a}$ & 25 \\
\hline H1N1/California/2009 & $<0.188$ & 1.563 & 1.563 & $>100$ or n/a \\
\hline H3N2/Nictoria/2011 & $<0.188$ & $>25$ or $\mathrm{n} / \mathrm{a}$ & $>50$ or $\mathrm{n} / \mathrm{a}$ & 6.25 \\
\hline H1N1/Michigan/2015 & $<0.125$ & 1.563 & 3.125 & $>12.5 \mathrm{or} \mathrm{n} / \mathrm{a}$ \\
\hline H1N1/Michigan/2017 & $<0.125$ & 25 & 25 & $>100$ or $\mathrm{n} / \mathrm{a}$
\end{tabular}

E

B

\begin{tabular}{|c|c|}
\hline & \\
\hline SERPS & QSADSIEXVP \\
\hline SSLQS & QQHASAPPYG \\
\hline SERPS & QSGDSTNRVV \\
\hline TMRPS & QSADSRGUVP \\
\hline SERPS & QSADSIDKGI \\
\hline TNKAS & QQNKELVPG \\
\hline SERPS & QSAMSINUANI \\
\hline SSLQS & QQHDSAPYG \\
\hline SERPS & QSADRIGSRI \\
\hline SERPS & QSVDrstarI \\
\hline TNEAS & FQGTQAPYG \\
\hline TNRPS & QSADSSDKVP \\
\hline SERPS & QSADSIDUSI \\
\hline SERPS & QSNDSIRWII \\
\hline SERPS & QSTDKSDKIP \\
\hline SERPS & QSADNTDPLI \\
\hline SSLQS & LQHSSAPYG \\
\hline NSRP? & ALWKCSAGDYYI \\
\hline SSLQS & FQHDNGYG \\
\hline SERPS & QSADSIDMAII \\
\hline SERPS & QSVDSIYKZI \\
\hline NNRPT & ALLGAP \\
\hline
\end{tabular}
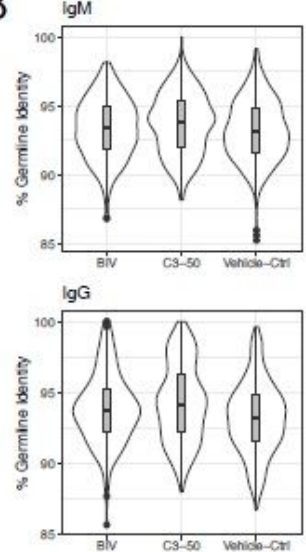 ER1-IMGT CDR1-IMGT ER2-IMGT CDR2-IMGT (39-55) (56-65)
FR3-IMGT (66-104)
LC
$(1-26)$
$[27-38\}$
50
60
$90 \quad 100$
CDR3-IMGT ER4-IMGI $(105-117) \quad(118-127)$

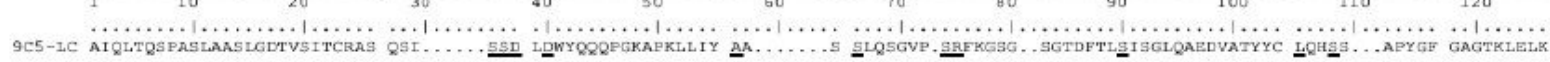

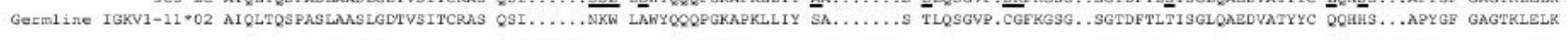

$\begin{array}{cccccc}\text { ER1-TMGT } & \text { CDR1-IMGT } & \text { TR2-IMGT } & \text { CDR2-IMGT } & \text { FR3-IMGT } & \text { CDR3-IMGT } \\ (1-26) & (27-38) & (39-55) & (56-65) & (66-104) & (105-117)\end{array}$

$\mathrm{HC}$

\begin{tabular}{llll}
\hline 1 & 10 & 20 & 30
\end{tabular}

60

80

90

100

110

120

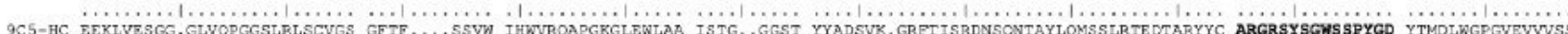

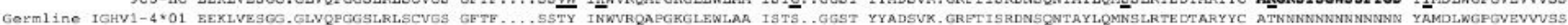

\section{Figure 4}

Conservation-Concentration Coupling induces uniform B cell expansion, affinity maturation, immunogenspecific and broadly neutralizing monoclonal antibodies A) Pig monoclonal antibodies specific to HA immunogen recovered from all C3 dosage groups (50ng/ag, 100ng/ag, 500ng/ag) of study \#1. Clones were recovered by isolating antibody repertoire DNA from PBMCs from all C3 immunized animals from study \#1, then selecting for anti-HA binder by phage display, and confirming individual clones by ELISA. B) Somatic hypermutation of top 100 most expanded clones in pigs, by isotype and immunization cohort.Immunization cohorts: a C3 formulation of $27 \mathrm{HAs}$ at 50ng/HA $(n=4)$, a bivalent cohort (H1N1 2007 and H3N2 2007 each at 5,000ng, $n=3$ ) and vehicle control (PBS + squalene, $n=$ 4). Lymphocytes collected 7 days after 6th vaccination (Day 112, study \#2). C) Percentage of peripheral B-cell receptor repertoire occupied by top $n$ clonotypes across pigs per immunization group. Gray: $95 \%$ confidence interval. D) Minimum antibody concentration $(\mathrm{ug} / \mathrm{mL})$ sufficient for broadly neutralizing pig 
monoclonal antibody 9C5 and controls CR9114, F10, C05 in-vitro neutralization of a panel of 5 Influenza A strains. Clone $9 \mathrm{C} 5$ was elicited by C3-50 immunized Pig \#25. E) Sequence alignment of broadly neutralizing pig monoclonal antibody 9 C5 to IMGT V Quest pig (Sus scrofa) germline genes. IMGT numbering. Detected somatic hypermutations underlined, $\mathrm{CDRH} 3$ marked in bold.

\section{Supplementary Files}

This is a list of supplementary files associated with this preprint. Click to download.

- NatureBiotechlvesGlanvilleMethods20201029.pdf 